\title{
Methods of RFID data processing in intelligent systems for the identification and movement control of industrial products
}

\author{
A V Astafiev ${ }^{1}$, A A Orlov ${ }^{1}$, D P Popov ${ }^{1}$ and M V Pshenichkin ${ }^{1}$ \\ ${ }^{1}$ Vladimir State University, Gorkij str. 87, Vladimir, Russia, 600000
}

\begin{abstract}
The article describes the development and research of methods of RFID data processing to build intelligent systems that provide timely and reliable automatic movement control and identification of industrial products. Conducted and presented an analytical review of Russian and foreign scientific-technical base for the development of methods and algorithms for movement control systems. The structure of the hardware-software complex of the system developed. The presented method of movement control products. Experimental studies of the developed system and methods.
\end{abstract}

\section{Introduction}

One of the most important elements of the quality management system of industrial production, which largely determines the efficiency of its functioning, is the mechanism of identification, which makes it possible to ensure traceability of products throughout the technological production cycle. Traceability in production helps to ensure compliance with government requirements and international quality standards, to perform a fast and targeted tracking of the entire technological cycle of manufacturing products, which, in turn, minimizes financial consequences. Especially relevant is the question of tracing products in the enterprise, if the production cycle consists of many stages, implemented in large production areas. The organization of the mechanism for tracing products is possible by automating the control of the movement of industrial products.

According to the GOST 18353-79 standard, there are 9 methods of non-destructive testing, of which 2 were widely used in the MCS: radio frequency identification and technical vision [1]. The use of technical vision approaches is complicated by the need for graphic marking of proper quality, which is difficult to realize in real production conditions and requires significant financial and human resources. The use of radio frequency identification is less demanding in the process of marking products. Traffic control, based on radio frequency identification methods, is an advanced information technology for the construction of warehouse accounting systems.

A large number of companies around the world are engaged in developing software and hardware for movement control systems (MCS), but the tasks of developing and implementing automated product tracking systems in production are still unresolved. At present, there are still a number of problems in industrial enterprises, the solution of which is not realized by means of modern MCS. These include:

- not all radio frequency markings may be in the field of view of equipment;

- a number of existing MCS can not be used in view of safety restrictions;

- not all MCS can ensure continuous and correct operation in large industrial areas;

- a number of modern MCS can work only in the presence of positioning systems, which are equipped with transporting devices; 
- the presence of interference and signals that make identification difficult.

The listed problems do not allow to organize automatic traffic control at all sections of the production process. To solve these problems, it is necessary to develop new methods that allow more efficient processing of RFID data.

Thus, the development of new methods for processing RFID data for the construction of automatic systems that provide an operative and reliable control over the movement of industrial products is topical.

The aim of the project is to develop and study methods for processing RFID data for the construction of intelligent systems that provide prompt and reliable automatic traffic control and identification of industrial products.

To achieve this goal, it is necessary to solve the following tasks:

1. Analytical review of the Russian and foreign scientific and technical base on development of methods and algorithms of MCS.

2. Development of the structure of the hardware-software complex MCS.

3. Development of an intelligent system and methods for controlling the movement of products.

4. Experimental research and modernization of the developed methods.

\section{Analytical review of the Russian and foreign scientific and technical base on the development of methods and algorithms MCS}

The PCS software is developed by: PCT-Invent (Russia, Sakt-Petersburg), AiTiProekt (Russia, Moscow), Impinj (USA, Seattle), Motorola (USA, Morrisville), Nordic ID (Finland, Salo), FEIG (Germany, Weilburg) and many others. However, the main activity of these companies is the development and production of the hardware part of radio frequency identification systems and the creation of specialized software for working with it. This implies that for the development of automatic identification systems based on the proposed software and hardware, it is necessary to collect a whole team of technical specialists to write the project "from scratch."

To quickly obtain real-time information on the location of the product and all its movements, systems based on the principles of radio frequency identification of product labels are used. The basis of the systems is the method of remote receipt of product data by transmitting a radio signal from the RFID tag located on its surface to the tags recognition devices (read) and then writing the information received to the database of computers forming the computer network. Such world-famous companies as Wal Mart, METRO Group, Gillette, Procter \& Gamble, Tesco, Benetton and others demonstrate on their practical experience the benefits of using RFID technology in the organization of automated warehouse accounting and cargo control.

The automatic identification system (AIS) of objects of rolling stock of railway transport (locomotives, cars, and also large-capacity containers) "Palma" is known, including a corporate computer network, code onboard sensors and reading points equipped at reference points, (RFID tags) the information on the location of the monitored object is automatically removed.

The disadvantage of this AAL Palma is the limited functionality of the system, which consists in the fact that information from the RFID tag is read only when the object passes the specially equipped information reading point (reader). Further location of the facility, for example in the warehouse, is not automatically recorded, which reduces the reliability and efficiency of using AIS when solving the problem of cargo storage.

The closest approach to the proposed approach is the JPL RFID system for warehouse accounting of metal pipes, based on the marking with radio frequency labels of plugs installed at the ends of pipes. This approach allows the inventory of stored products in an automated mode using hand-held portable readers.

The disadvantage of this AIS is that the movement of pipe products is produced by various means of minor mechanization in such a way that the marking installed on the product in most cases does not fall within the field of view of the reading sensors, which makes it impossible to track the products in automatic mode.

A common disadvantage for this kind of AIS is that for the organization of automatic traffic control, readers are installed permanently, and the movement of the tracked products occurs as it 
passes along this device. An obligatory requirement is that the marking is in line of sight for the reader, which is practically impossible with the use of such transport devices as bridge cranes, loaders, stackers and other means of minor mechanization. The movement of products for this type of transportation is of a stochastic nature, and the location of the cargo can close the direct visibility of the reader to the marking.

The scientific community is also working on the introduction of RFID technologies in various spheres of human activity. A great contribution to the development of radio frequency identification technology and SKD in various spheres of life was made by Bondarevsky AS, Zolotov RV, Do Zuy Nyat, Kamozin D.Yu., Manish B., Shahram M., Ke-Sheng Wang, Worapot Jakkhupan, Somjit Archint, Yuefeng Li, Mahir Oner, Alp Ustundag, Aysenur Budak and many others. The latest works in this area are [2-7].

The application of these knowledge-intensive technologies makes it possible to automate the processes of controlling the movement of industrial products at enterprises of various spheres of life and, ultimately, to improve the efficiency and reliability of the control of transportation and warehouse accounting of manufactured products.

However, they are not without flaws. The use of existing software and hardware solutions is more focused on the organization of automated warehouse accounting and is less suitable for automating traffic control. In confirmation of this at a number of industrial enterprises.

\section{Development of the structure of the hardware and software complex MCS}

The development of intellectual MCS is aimed at solving the task of organizing and providing control over the movement of products through the territory of industrial enterprises, handling and cargo transportation, including warehousing of goods equipped with multi-code marking. Information on the identification of products can be collected in the database of computers that are part of the computer network that encompasses the points of the cargo transportation route and stores data on the origin and destination of the products and on its location in warehouses.

The proposed MCS consists of 4 main modules: server, client, product marking and marking of the storage area.

Marking of storage areas is performed using Bluetooth-labels, and the current location of the smallscale mechanization means is determined using the IBeacon technology.

Labelling of products at the enterprise is done by attaching an RFID tag to them. In the event that the product contains components shielding the signal from the mark, the marking is applied to several sides of the product.

The client is a stand-alone device equipped with an RFID reader to collect data from RFID tags of products, a Bluetooth adapter for determining the current location, a microcomputer for collecting, processing and sending telegrams to the server and batteries to provide autonomous operation.

The server is a computing device that receives telegrams from client devices, compares the received data with information from the database and generates messages about movements transmitted to the server of the plant's process control system.

The structure of the hardware-software complex of the MCS being developed is shown in Figure 1.

The developed architecture is applicable to various means of minor mechanization. Figure 2 shows an example of placement on a bridge crane.

The proposed system is explained with the following drawing (figure 2), on which it is indicated:

1 - Bridge crane, for moving products;

2 - Bridge crane truck;

3 - Bridge crane beams;

4 - Movable cargo or article;

5 - Customer;

6 - Product identification;

7 - Warehouse area limiter;

8 - Marking of the storage area. 


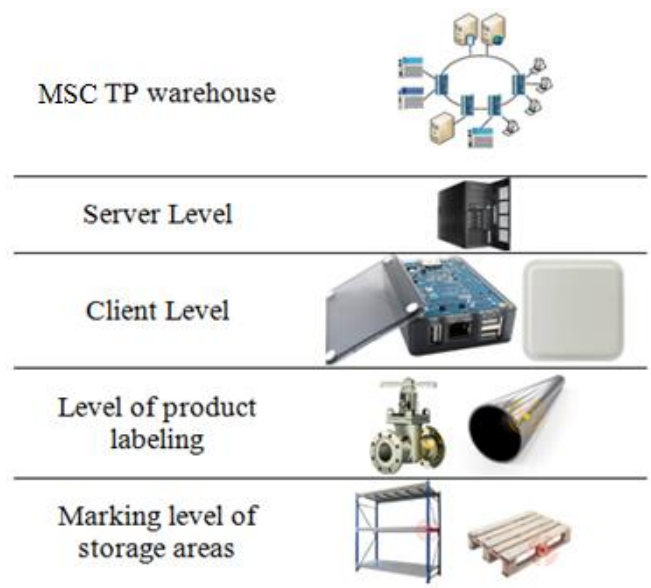

Figure 1. The structure of the hardware and software complex of the developed MCS.

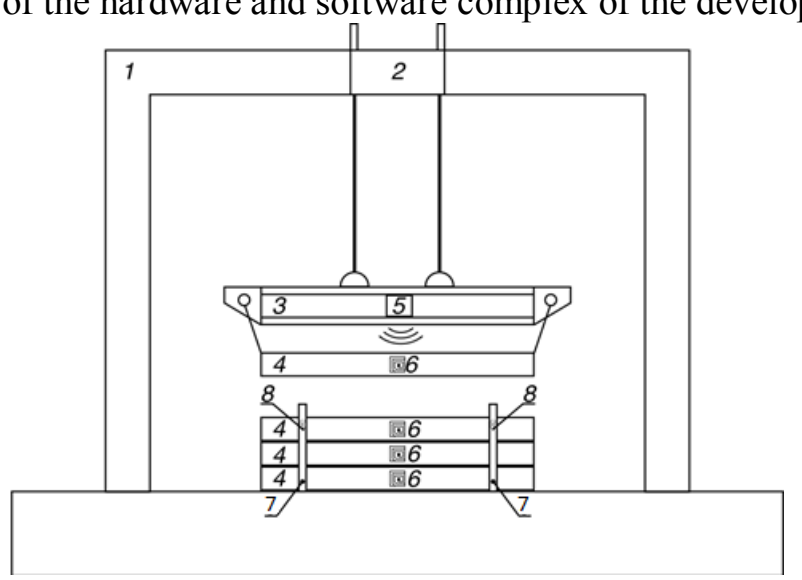

Figure 2. General scheme of means of moving pipes.

\section{Development of an algorithm for determining the threshold for clipping markers that are not involved in the movement}

The aim of the work is to develop an algorithm for filtering RFID tags, which makes it possible to exclude marks that are not involved in the course of movement and to conduct a series of laboratory tests of the developed system for automatic control over the movement of products along untyped routes.

A disturbance is an external perturbation acting in the transmission system and preventing proper reception of signals. Sources of interference may be either outside or inside the transmission system itself. If the interference is regular and known, then fighting it is not difficult. For example, the background of an alternating current can be eliminated by compensation; interference from a particular radio station with a modulation spectrum of normal width is eliminated by an appropriate filter. The struggle with random interference presents the greatest difficulty.

There are many methods of detecting interference. The following were considered in the work:

1. The arithmetic mean.

2. Determination of emissions in statistics.

3. Determination of the optimum noise level in the flow of spectra.

4. Level of significance.

Two shelves have been created, each of which is marked with an iBeacon-tag. Portable products are marked with RFID tags. Moves three products 1, 3, 4 from the first rack to the second one. Items c labeled 2 and 5 are located between the shelves and are not subjected to transfer. Table 1 presents the primary data.

During the move, 23 signal level values (RSSI) were received from all RFID tags.

To determine the threshold value of $\mathrm{p}$, which will allow us to weed out marks that are not involved in the movement, four methods were tested (Figure $3 \mathrm{a}-\mathrm{d}$ ). 
Table 1. Results of displacement.

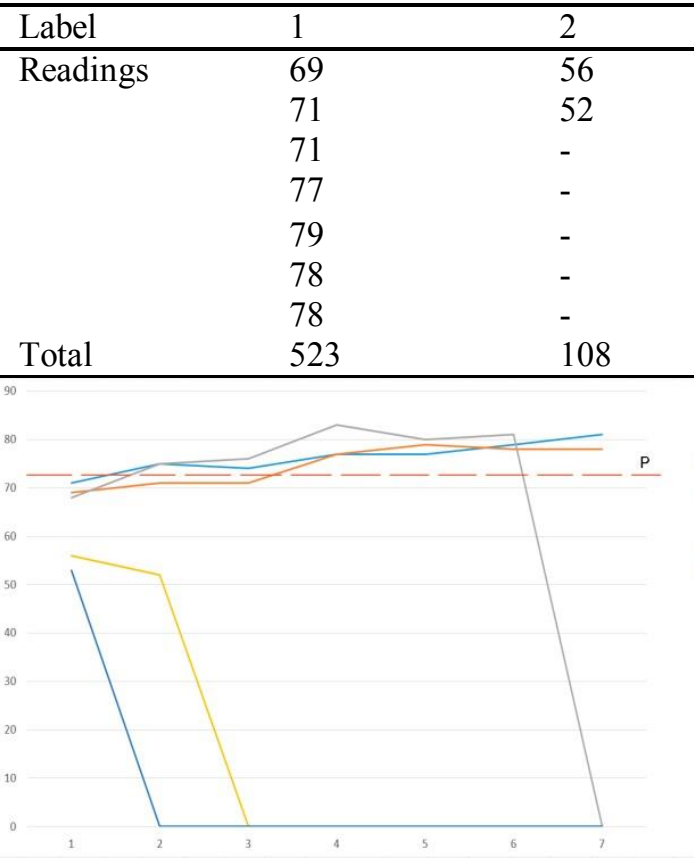

a)

\begin{tabular}{ll}
4 & 5 \\
\hline 68 & 53 \\
75 & - \\
76 & - \\
83 & - \\
80 & - \\
81 & - \\
- & - \\
463 & 53 \\
\hline
\end{tabular}

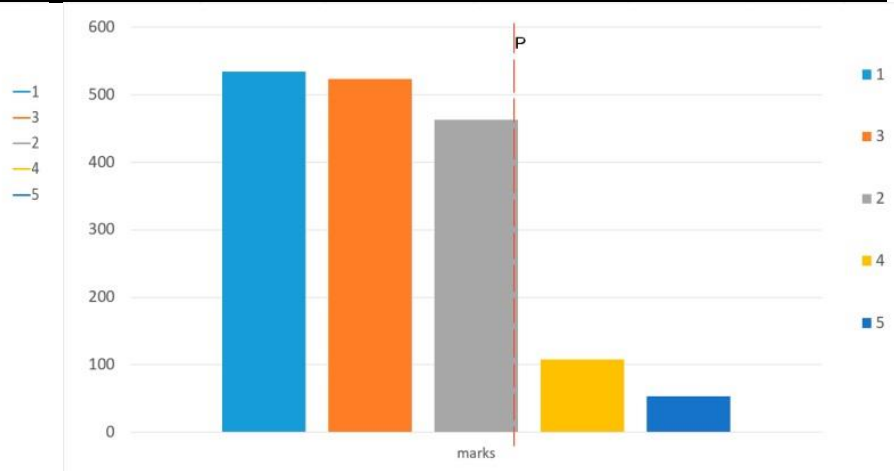

b)

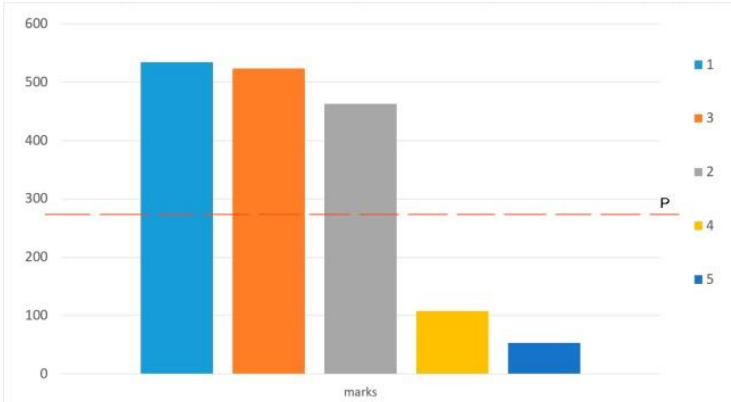

c)

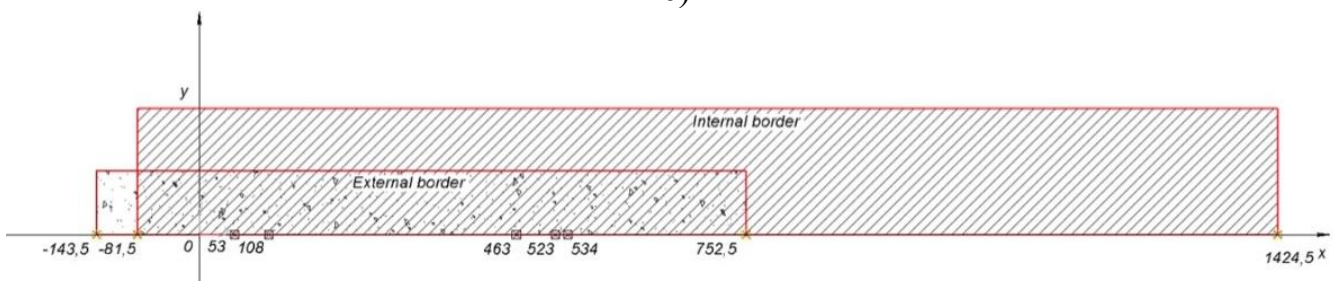

d)

Figure 3. a) Allocation of useful data on the threshold $P$, based on the arithmetic mean, b) Determination of useful data on the histogram, c) Determination of the threshold by significance level $\mathrm{p}=0.10, \mathrm{~d})$ Determination of emissions.

Laboratory investigations were carried out, during which it was established that the method based on the level of significance was the most suitable method for testing the tested methods. The determination of noise by percentage of the area or the arithmetic mean does not always yield correct results. The emission detection method can not be used for this task, since false signals are not discarded, due to the small sample that is obtained in most cases.

\section{Experimental results}

To test the evaluation of the reliability of the results obtained, an experimental study was carried out. The subject of the study is a system for automatic control over the movement of products. The object is a product, an enterprise or a warehouse. 
Laboratory experience was conducted with a prototype system in conditions close to real production. To the moving cart was attached a model of the part of the beam, with the reading and processing device mounted on the side. Under the traverse on the cart is mounted a pallet for products in the form of pipes. View of the laboratory setup is shown in figure 4.

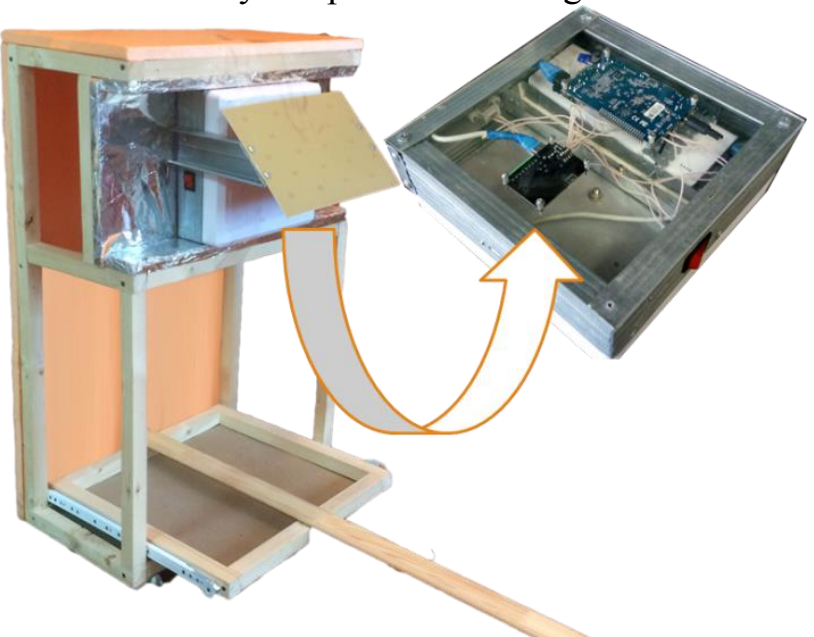

Figure 4. View of the laboratory setup.

For the experiment was created two racks $(\mathrm{c} 1, \mathrm{c} 2)$, each of which is labelled iBeacon-tag. The movement of products made from rack 1 to rack 2. During the movement of production readings from their RFID-tags and iBeacon-tags of racks have been processed and are presented in tables (Table 2, $3)$.

Table 2. Readings from iBeacon-tags of racks.

\begin{tabular}{lll}
\hline Time & Rack 1 & Rack 2 \\
\hline $35: 22: 00$ & 0 & 43 \\
$35: 28: 00$ & 0 & 26 \\
$35: 34: 00$ & 0 & 0 \\
$35: 40: 00$ & 0 & 0 \\
$35: 46: 00$ & 3 & 0 \\
$35: 52: 00$ & 34 & 0 \\
$35: 58: 00$ & 37 & 0 \\
$36: 04: 00$ & 27 & 0 \\
$36: 10: 00$ & 27 & 0 \\
$36: 16: 00$ & 16 & 0 \\
\hline
\end{tabular}

Table 3. Readings from RFID tags of products.

\begin{tabular}{lrrrrr}
\hline Time & Product 1 & Product 2 & Product 3 & Product 4 \\
\hline $35: 22: 00$ & 65 & 16 & 91 & 89 \\
& 61 & 5 & 101 & 101 \\
$35: 28: 00$ & 75 & 20 & 105 & 105 \\
$35: 00$ & 81 & 11 & 108 & 107 \\
$35: 46: 00$ & 73 & 9 & 110 & 109 \\
$35: 52: 00$ & 57 & 21 & 98 & 95 \\
$35: 58: 00$ & 38 & 7 & 103 & 110 \\
$36: 04: 00$ & 38 & 21 & 99 & 110 \\
$36: 10: 00$ & 61 & 2 & 107 & 110 \\
$36: 16: 00$ & 35 & 3 & 78 & 80 \\
\hline
\end{tabular}

The duration of the experiment was 1 minute. During the experiment with iBeacon-tags of rack has received 212 readings and from RFID tags of products - 2715. Graphic interpretation of the data presented in figures 5,6 . 


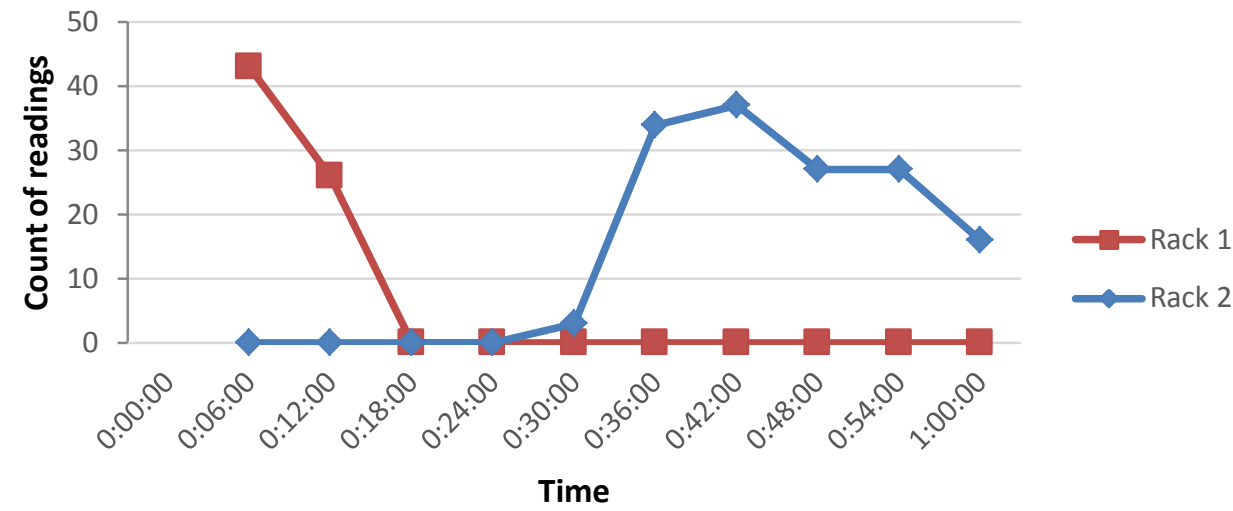

Figure 5. Readings from iBeacon-tags of racks.

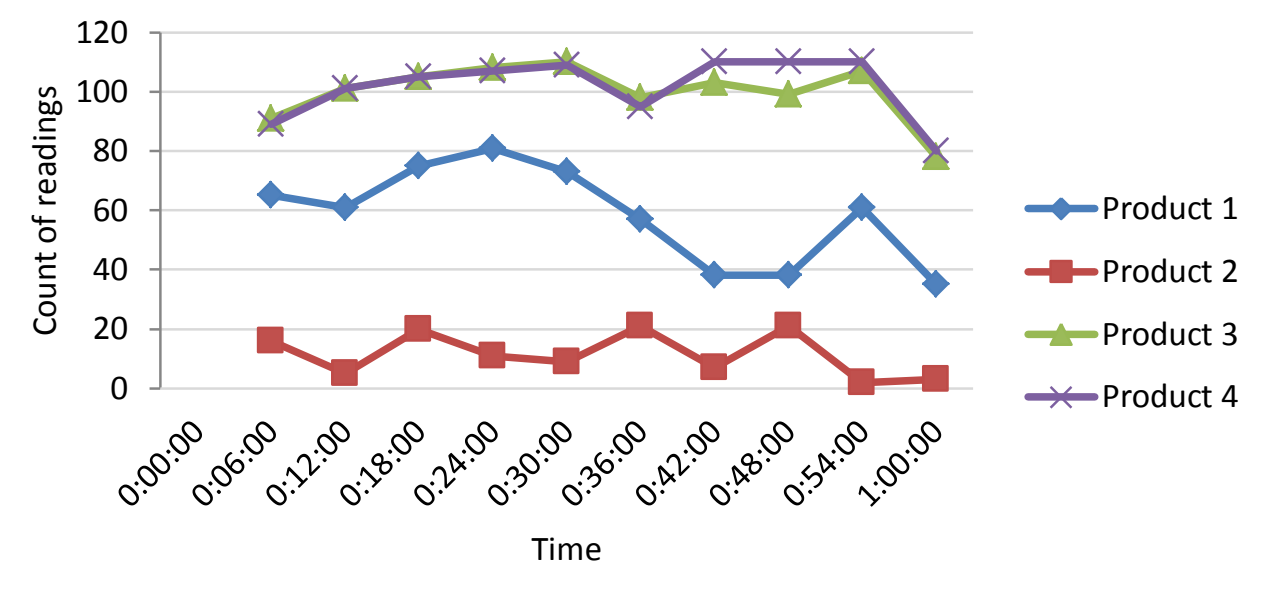

Figure 6. Readings from RFID tags of products.

From the graph in figure 4 shows that at time 00:18:00 readings from iBeacon-tag of the first rack were no longer received by the reader. At time 0:24:00 began to receive readings from iBeacon-tag of the rack number 2 . This fact indicates that there was a movement of the transport device from the area of the rack 1 to the area of the rack 2 .

Figure 5 shows the readings from the RFID-tags of products, time-spaced with an interval of 6 seconds. During the experiment, 4 items were moved. According to the graph it is clear that a stable signal came from all 4 product tags. This allows us to say that during the movement of the conveying device the products moved along with it.

In the course of the work, more than 150 experiments were conducted in the laboratory. Also, the installation was tested in an industrial plant. The results of the experiment showed the reliability of the movement identifications in the amount of $97.3 \%$. As a result, information was also collected that allowed adjustments to the system to improve its efficiency.

\section{Conclusion}

A system and method for automatic control of the movement of products based on RFID-identification when moving hoisting-and-transport mechanisms is developed. Considered and analyzed the existing systems of automatic control of the movement of products based on radio frequency identification. The main advantages and disadvantages are revealed. The structure and levels of the hardware and software complex MCS are described, the methodology of its operation is described. Thanks to the presented system and methodology, the problems of moving several units of products simultaneously were solved, the accuracy of detail of the location information was high, the client-server approach and the movement history analysis algorithms were implemented, the problem of authenticity of 
identification and the tracking process outside the route was solved. Laboratory investigations were conducted, during which it was possible to obtain a high degree of reliability of the identification of displacements in $97.3 \%$, proving the urgency of the developed system and methodology. These studies bear a high scientific value for providing control over the movement of products.

\section{References}

[1] GOST 18353-79 Nondestructive check. Classification of types and methods

[2] Astafiev A V, Orlov A A and Popov D P 2017 Development the algorithm of positioning industrial wares in-plant based on radio frequency identification for the products tracking systems CEUR Workshop Proceeding 1901 23-27

[3] Astafiev A V, Orlov, A A and Privezencev D G 2016 Method of controlling the movement of large metal products with the use of algorithms for localization and recognition of bar code markings Dynamics of Systems, Mechanisms and Machines, Dynamics 7819046 DOI: 10.1109/Dynamics.2016.7818969

[4] Orlov A A, Provotorov A V and Astafiev A V 2016 Methods and algorithms of automated twostage visual recognition of metal-rolling billets Automation and Remote Control 1099-1105 DOI: $10.1134 /$ S000511791606014X

[5] Zhiznyakov A L, Privezentsev D G and Zakharov A A 2015 Using fractal features of digital images for the detection of surface defects Pattern Recognition and Image Analysis 25(1) 122-131

[6] Nhat D D 2015 Researches and application of RFID technology (radio frequency identification) International Research Journal 5 34-37

[7] Kamozin D Y 2013 Comparison of the effectiveness of bar-code technology and RFID technology's application in logistics processes Bulletin of Baikal State University 3 71-75

[8] Parikh D and Jancke G 2008 Localization and segmentation of a 2D high capacity color barcode Proceeding of IEEE Workshop on Applications of Computer Vision 1-6 DOI: 10.1109/WACV.2008.4544033

[9] Kazanskiy N L and Popov S B 2012 The distributed vision system of the registration of the railway train Computer Optics 36(3) 419-428

[10] Morozov A A and Sushkova O S 2016 Analysis of real-time video images using the means of the Actor Prologue Computer Optics 40(6) 947-957 DOI: 10.18287 / 2412-6179-2016-40-6947-957

[11] Epifantsev B N, Pyatkov A A and Kopeikin S A 2016 Multisensory systems for monitoring restricted areas: the capabilities of a video analytics channel for intrusion detection Computer Optics 40(1) 121-129 DOI: 10.18287 / 2412-6179-2016-40-1-121-129 